\title{
Estimation of the Weibull tail-coefficient with linear combination of upper order statistics
}

\author{
Laurent Gardes \& Stéphane Girard \\ INRIA Rhône-Alpes, projet Mistis, Inovallée, 655, av. de l'Europe, \\ Montbonnot, 38334 Saint-Ismier cedex, France. \\ \{Laurent.Gardes, Stephane.Girard\}@inrialpes.fr
}

\begin{abstract}
We present a new family of estimators of the Weibull tail-coefficient. The Weibull tail-coefficient is defined as the regular variation coefficient of the inverse failure rate function. Our estimators are based on a linear combination of log-spacings of the upper order statistics. Their asymptotic normality is established and illustrated for two particular cases of estimators in this family. Their finite sample performances are presented on a simulation study.
\end{abstract}

Keywords: Weibull tail-coefficient, extreme-values, order statistics, regular variations.

\section{Introduction}

Weibull tail-distributions encompass a variety of light tailed distributions, i.e. distributions in the Gumbel maximum domain of attraction, see [12 for further details. Weibull taildistributions include for instance Weibulls, Gaussians, gammas and logistic. The purpose of this paper is to study the estimation of a tail parameter associated with these distributions. More precisely, a cumulative distribution function $F$ has a Weibull tail if its logarithmic tail satisfies the following property: There exists $\theta>0$ such that for all $\lambda>0$,

$$
\lim _{t \rightarrow \infty} \frac{\log (1-F(\lambda t))}{\log (1-F(t))}=\lambda^{1 / \theta} .
$$

The parameter of interest $\theta$ is called the Weibull tail-coefficient. Such distributions are of great use to model large claims in non-life insurance [5]. In the particular case where $\log (1-F(\lambda t)) / \log (1-F(t))=\lambda^{1 / \theta}$ for all $t>0$ and $\lambda>0$, estimating $\theta$ reduces to estimating the shape parameter of a Weibull distribution. In this context, simple and efficient methods exist, see for instance [2], Chapter 4 for a review on this topic. Otherwise, dedicated estimation methods have been proposed since the relevant information on the Weibull tail-coefficient is only contained in the extreme upper part of the sample. A first direction was investigated in [7] where an estimator based on the record values is proposed. Another family of approaches [9, 4, 6, 16, consists of using the $k_{n}$ upper order statistics where $\left(k_{n}\right)$ is an intermediate sequence of integers i.e. such that

$$
\lim _{n \rightarrow \infty} k_{n}=\infty \text { and } \lim _{n \rightarrow \infty} k_{n} / n=0 .
$$

Note that, since $\theta$ is defined only by an asymptotic behavior of the tail, the estimator should use the only extreme-values of the sample and thus the second part of (2) is required. The estimators considered here belong to this approach. Let $\left(X_{i}\right)_{1 \leq i \leq n}$ be a sequence of independent and identically distributed random variables with cumulative distribution function $F$. 
Denoting by $X_{1, n} \leq \ldots \leq X_{n, n}$ the corresponding order statistics, our family of estimators is $\hat{\theta}_{n}(\alpha)=\sum_{i=1}^{k_{n}-1} \alpha_{i, n}\left(\log \left(X_{n-i+1, n}\right)-\log \left(X_{n-k_{n}+1, n}\right)\right) / \sum_{i=1}^{k_{n}-1} \alpha_{i, n}\left(\log \log (n / i)-\log \log \left(n / k_{n}\right)\right)$

with weights $\alpha_{i, n}=W\left(i / k_{n}\right)+\varepsilon_{i, n}$ defined from $W$ a smooth score function and $\left(\varepsilon_{i, n}\right)_{1 \leq i \leq k_{n}-1}$ a non-random sequence. We refer to [10, 23] for similar works in the context of the estimation of the extreme-value index.

In Section 2 we state the asymptotic normality of these estimators. In Section 3 we provide two examples of weights. The first one leads to the estimator of $\theta$ proposed by Beirlant et al. [6]. The second one gives rise to a new estimator for Weibull tail-distributions. The behavior of these two estimators is investigated on finite sample situations in 14. Finally, proofs are given in Section 4

\section{Asymptotic normality}

Consider the failure rate $H=-\log (1-F)$. Writing $H^{\leftarrow}$ its generalized inverse $H^{\leftarrow}(t)=$ $\inf \{x, H(x) \geq t\}$, assumption (11) is equivalent to:

(A.1) $H^{\leftarrow}(t)=t^{\theta} \ell(t)$,

where $\ell$ is a slowly varying function i.e. such that $\ell(\lambda t) / \ell(t) \rightarrow 1$ as $t \rightarrow \infty$ for all $\lambda>0$. The inverse failure rate function $H^{\leftarrow}$ is said to be regularly varying at infinity with index $\theta$ and this property is denoted by $H^{\leftarrow} \in \mathcal{R}_{\theta}$. We refer to [8] for more information on regular variation theory. As a comparison, Pareto type distributions satisfy $(1 /(1-F))^{\leftarrow} \in \mathcal{R}_{\gamma}$, and $\gamma>0$ is the so-called extreme-value index. As often in extreme-value theory, (A.1) is not sufficient to prove a central limit theorem for $\hat{\theta}_{n}(\alpha)$. It needs to be strengthened with a second order condition on $\ell$, namely that there exist $\rho \leq 0$ and a function $b$ with limit 0 at infinity such that

(A.2) $\log (\ell(\lambda t) / \ell(t)) \sim b(t) \int_{1}^{\lambda} u^{\rho-1} d u$,

uniformly locally on $\lambda>1$ and as $t \rightarrow \infty$. The second order parameter $\rho \leq 0$ tunes the rate of convergence of $\ell(\lambda t) / \ell(t)$ to 1 . The closer $\rho$ is to 0 , the slower is the convergence. Condition (A.2) is the cornerstone in all proofs of asymptotic normality for extreme-value estimators. It is used in [18, 17, 3] to prove the asymptotic normality of estimators of the extremevalue index $\gamma$. Table 1 shows that many distributions satisfy (A.1) and (A.2). Among them, Extended Weibull distributions, introduced in [19], encompass gamma, Gaussian and Benktander II distributions. We refer to [11, Table 3.4.4, for the derivation of $b(x)$ and $\rho$ in each case. Other examples are the Weibull, logistic and extreme-value (with shape parameter $\gamma=0)$ distributions.

Throughout the paper, we write Id for the identity function. In particular, if $f$ is a function and $p$ a real number, the inequality $f \leq \operatorname{Id}^{p}$ means $f(t) \leq t^{p}$ for any $p$ where it is defined. For general L-estimators, conditions on the weights are required to obtain a central limit theorem (see for instance [21]). Our assumptions are the following:

(A.3) $W$ is defined and continuously differentiable on the open unit interval,

(A.4) There exist $M>0,0 \leq q<1 / 2$ and $p<1$ such that $|W| \leq M \operatorname{Id}^{-q}$ and $\left|W^{\prime}\right| \leq$ $M \mathrm{Id}^{-p-q}$ on the open unit interval.

Similar conditions have been introduced in the context of the estimation of the extreme-value index [10, 23]. To write the limiting variance of $\hat{\theta}_{n}(\alpha)$, we introduce two quantities:

$$
\begin{aligned}
\mu(W) & =\int_{0}^{1} W(x) \log (1 / x) d x \\
\sigma^{2}(W) & =\int_{0}^{1} \int_{0}^{1} W(x) W(y) \frac{\min (x, y)-x y}{x y} d x d y .
\end{aligned}
$$


We also define $\|\varepsilon\|_{n, \infty}=\max _{i=1, \ldots, k_{n}-1}\left|\varepsilon_{i, n}\right|$. We are now in position to state our main result. Its proof is postponed to Section 4

Theorem 1 Suppose (A.1)-(A.4) hold. If $\left(k_{n}\right)$ is any intermediate sequence such that

$$
k_{n}^{1 / 2} b(\log (n)) \rightarrow \lambda \text { and } k_{n}^{1 / 2} \max \left\{1 / \log (n),\|\varepsilon\|_{n, \infty}\right\} \rightarrow 0,
$$

then

$$
k_{n}^{1 / 2}\left(\hat{\theta}_{n}(\alpha)-\theta\right) \stackrel{d}{\rightarrow} \mathcal{N}\left(\lambda, \theta^{2} \sigma^{2}(W) / \mu^{2}(W)\right) .
$$

Clearly, the bias of the estimator is driven by the function $b$. This bias term asymptotically vanishes if $\lambda=0$. Some applications of this result are given in the next section, Corollary 1 and Corollary 2, The importance of the bias term is also illustrated on finite sample situations. Finally, note that condition (4) implies $k_{n} / n \rightarrow 0$.

\section{Comparison of two estimators}

First, we show in Paragraph 3.1, that our family of estimators (3) encompasses the Hill type estimator $\hat{\theta}_{n}^{H}$ proposed in [6]. Moreover, it will appear in Corollary 1 that the asymptotic normality of $\hat{\theta}_{n}^{H}$ stated in [16], Theorem 2 is a consequence of our main result Theorem 1. Second, in Paragraph 3.2, we use our framework to exhibit a new estimator of the Weibull tailcoefficient and to establish its asymptotic normality in Corollary 2. In the third paragraph, we show that the new estimator performs as well as the Hill one.

\subsection{Hill type estimator}

Beirlant et al. [16] propose the following estimator of the Weibull tail-coefficient:

$$
\hat{\theta}_{n}^{H}=\sum_{i=1}^{k_{n}-1}\left(\log \left(X_{n-i+1, n}\right)-\log \left(X_{n-k_{n}+1, n}\right)\right) / \sum_{i=1}^{k_{n}-1}\left(\log \log (n / i)-\log \log \left(n / k_{n}\right)\right) .
$$

Clearly, $\hat{\theta}_{n}^{H}$ is a particular case of $\hat{\theta}_{n}(\alpha)$ with $W(x)=1$ for all $x \in[0,1]$ and $\varepsilon_{i, n}=0$ for all $i=1, \ldots, k_{n}$. The asymptotic normality of $\hat{\theta}_{n}^{H}$, established in Theorem 2 of [16], can be obtained as a consequence of Theorem 1

Corollary 1 Suppose (A.1) and (A.2) hold. If $\left(k_{n}\right)$ is an intermediate sequence such that $k_{n}^{1 / 2} \max \{b(\log (n)), 1 / \log (n)\} \rightarrow 0$, then $k_{n}^{1 / 2}\left(\hat{\theta}_{n}^{H}-\theta\right) \stackrel{d}{\rightarrow} \mathcal{N}\left(0, \theta^{2}\right)$.

\subsection{Zipf estimator}

We propose a new estimator of the Weibull tail-coefficient based on a quantile plot adapted to our situation. It consists of drawing the pairs $\left(\log \log (n / i), \log \left(X_{n-i+1, n}\right)\right)$ for $i=1, \ldots, n-1$. The resulting graph should be approximatively linear (with slope $\theta$ ), at least for the large values of $i$. Thus, we introduce $\hat{\theta}_{n}^{Z}$ the least square estimator of $\theta$ based on the $k_{n}$ largest observations:

$$
\hat{\theta}_{n}^{Z}=\sum_{i=1}^{k_{n}-1}\left(\log \log (n / i)-\zeta_{n}\right) \log \left(X_{n-i+1, n}\right) / \sum_{i=1}^{k_{n}-1}\left(\log \log (n / i)-\zeta_{n}\right) \log \log (n / i),
$$

where

$$
\zeta_{n}=\frac{1}{k_{n}-1} \sum_{i=1}^{k_{n}-1} \log \log (n / i) .
$$

This estimator is similar to the Zipf estimator for the extreme-value index proposed by Kratz and Resnick 20] and Schultze and Steinebach [22. We prove in Section 4 that $\hat{\theta}_{n}^{Z}$ belongs to family (3) and thus apply Theorem 1 to obtain its asymptotic normality: 
Corollary 2 Suppose (A.1) and (A.2) hold. If $\left(k_{n}\right)$ is an intermediate sequence such that $k_{n}^{1 / 2} \max \left\{b(\log (n)), \log ^{2}\left(k_{n}\right) / \log (n)\right\} \rightarrow 0$, then $k_{n}^{1 / 2}\left(\hat{\theta}_{n}^{Z}-\theta\right) \stackrel{d}{\rightarrow} \mathcal{N}\left(0,2 \theta^{2}\right)$.

\section{Proofs}

Throughout this section, we assume that $\left(k_{n}\right)$ is an intermediate sequence and, for the sake of simplicity, we note $k$ for $k_{n}$. Let us also introduce $K_{\rho}(\lambda)=\int_{1}^{\lambda} u^{\rho-1} d u$ for $\lambda \geq 1$ and $J(x)=W(1-x)$ for $x \in(0,1)$. The following notations will prove useful: $E_{n-k+1, n}$ is the $(n-k+1)$ th order statistics associated to $n$ independent standard exponential variables and $\left(F_{i, k-1}\right)_{1 \leq i \leq k-1}$ are order statistics, independent from $E_{n-k+1, n}$, generated by $k-1$ independent standard exponential variables. The next lemma presents an expansion of $\hat{\theta}_{n}(\alpha)$.

Lemma 1 Under (A.1) and (A.2), $\hat{\theta}_{n}(\alpha)$ has the same distribution as

$$
\frac{\theta T_{n}^{(2,0)}+\left(1+o_{P}(1)\right) b\left(E_{n-k+1, n}\right) T_{n}^{(2, \rho)}}{T_{n}^{(1)}}
$$

where we have defined

$$
\begin{aligned}
T_{n}^{(1)} & =\frac{1}{k-1} \sum_{i=1}^{k-1} \alpha_{i, n}(\log \log (n / i)-\log \log (n / k)) \text { and } \\
T_{n}^{(2, \rho)} & =\frac{1}{k-1} \sum_{i=1}^{k-1} \alpha_{k-i, n} K_{\rho}\left(1+\frac{F_{i, k-1}}{E_{n-k+1, n}}\right), \rho \leq 0 .
\end{aligned}
$$

Proof : Using the quantile transform, the order statistics $\left(X_{i, n}\right)_{1 \leq i \leq n}$ have the same distribution as $\left(H^{\leftarrow}\left(E_{i, n}\right)\right)_{1 \leq i \leq n}$. Thus, (A.2) yields that the numerator of $\hat{\theta}_{n}(\alpha)$ in (3) has the same distribution as

$$
\theta \frac{1}{k-1} \sum_{i=1}^{k-1} \alpha_{i, n} \log \left(\frac{E_{n-i+1, n}}{E_{n-k+1, n}}\right)+\left(1+o_{P}(1)\right) b\left(E_{n-k+1, n}\right) \frac{1}{k-1} \sum_{i=1}^{k-1} \alpha_{i, n} K_{\rho}\left(\frac{E_{n-i+1, n}}{E_{n-k+1, n}}\right) .
$$

The Rényi representation asserts that $\left(E_{n-i+1, n} / E_{n-k+1, n}\right)_{1 \leq i \leq k-1}$ has the same distribution as $\left(1+F_{k-i, k-1} / E_{n-k+1, n}\right)_{1 \leq i \leq k-1}$, see [1], p. 72. Therefore, the numerator of $\hat{\theta}_{n}(\alpha)$ has the same distribution as

$$
\begin{aligned}
& \theta \frac{1}{k-1} \sum_{i=1}^{k-1} \alpha_{i, n} \log \left(1+\frac{F_{k-i, k-1}}{E_{n-k+1, n}}\right) \\
+\quad & \left(1+o_{P}(1)\right) b\left(E_{n-k+1, n}\right) \frac{1}{k-1} \sum_{i=1}^{k-1} \alpha_{i, n} K_{\rho}\left(1+\frac{F_{k-i, k-1}}{E_{n-k+1, n}}\right) .
\end{aligned}
$$

Changing $i$ to $k-i$ in the above formula and remarking that $K_{0}$ is the logarithm function conclude the proof.

The following lemma provides an expansion of

$$
\tau_{n}=\frac{1}{k-1} \sum_{i=1}^{k-1}(\log \log (n / i)-\log \log (n / k)),
$$

which frequently appears in the proofs.

Lemma 2 The following expansion holds:

$$
\tau_{n}=\frac{1}{\log (n / k)}\left\{1+O\left(\frac{\log (k)}{k}\right)+O\left(\frac{1}{\log (n / k)}\right)\right\} .
$$


Proof : We write $\tau_{n}$ as the sum

$$
\frac{1}{\log (n / k)} \frac{1}{k-1} \sum_{i=1}^{k-1} \log (k / i)+\frac{1}{k-1} \sum_{i=1}^{k-1}\left\{\log \left(1+\frac{\log (k / i)}{\log (n / k)}\right)-\frac{\log (k / i)}{\log (n / k)}\right\} .
$$

Since

$$
\frac{1}{k-1} \sum_{i=1}^{k-1} \log (i / k)=\frac{1}{k-1} \log \left(\frac{k !}{k^{k}}\right)
$$

Stirling's formula shows that the first term is

$$
\frac{1}{\log (n / k)}\left(1+O\left(\frac{\log (k)}{2 k}\right)\right) \text {. }
$$

The inequality $-x^{2} / 2 \leq \log (1+x)-x \leq 0$, valid for nonnegative $x$ shows that the second term is of order at most

$$
\frac{1}{\log ^{2}(n / k)} \frac{1}{k-1} \sum_{i=1}^{k-1} \log ^{2}(i / k)=O\left(\frac{1}{\log ^{2}(n / k)}\right)
$$

since the above Riemann sum converges to 2 as $k \rightarrow \infty$. The result follows.

The next lemmas are dedicated to the study of the different terms appearing in Lemma 1 , First, we focus on the non-random term $T_{n}^{(1)}$.

Lemma 3 Under (A.1)-(A.4), the following expansion hold:

$$
T_{n}^{(1)}=\frac{\mu(W)}{\log (n / k)}\left\{1+O\left(\log (k) k^{q-1}\right)+O\left(\frac{1}{\log (n / k)}\right)+O\left(\|\varepsilon\|_{n, \infty}\right)\right\} .
$$

Proof : Clearly, $T_{n}^{(1)}$ can be rewritten as the sum

$$
\frac{1}{k-1} \sum_{i=1}^{k-1} \varepsilon_{i, n} \log \left(1+\frac{\log (k / i)}{\log (n / k)}\right)+\frac{1}{k-1} \sum_{i=1}^{k-1} W(i / k) \log \left(1+\frac{\log (k / i)}{\log (n / k)}\right) .
$$

The absolute value of the first term is less than $\|\varepsilon\|_{n, \infty} \tau_{n}$ which is $O\left(\|\varepsilon\|_{n, \infty} / \log (n / k)\right)$, by Lemma 2. The second term can be expanded as

$$
\begin{array}{r}
\frac{1}{\log (n / k)} \frac{1}{k-1} \sum_{i=1}^{k-1} W(i / k) \log (k / i)+\frac{1}{k-1} \sum_{i=1}^{k-1} W(i / k)\left\{\log \left(1+\frac{\log (k / i)}{\log (n / k)}\right)-\frac{\log (k / i)}{\log (n / k)}\right\} \\
=: \frac{T_{n}^{(1,1)}}{\log (n / k)}+T_{n}^{(1,2)} .
\end{array}
$$

For $x \in(0,1)$, define $H(x)=W(x) \log (1 / x)$. The Riemann sum $T_{n}^{(1,1)}$ can be compared to $\mu(W)$ by:

$$
\left|T_{n}^{(1,1)}-\mu(W)\right| \leq \frac{1}{2 k^{2}} \sum_{i=1}^{k-1} \sup _{i / k \leq x \leq(i+1) / k}\left|H^{\prime}(x)\right|+\int_{0}^{1 / k}|H(x)| d x+O(1 / k) .
$$

Assumption (A.4) implies that there exists a positive $M^{\prime}$ such that $\left|H^{\prime}\right| \leq M^{\prime} \mathrm{Id}^{-q-1}$ on the open unit interval, and thus the first term of (7) is bounded above by

$$
\frac{M^{\prime}}{2 k}\left(\int_{1 / k}^{1} t^{-q-1} d t+k^{q}\right)= \begin{cases}O\left(k^{q-1}\right) & \text { if } q \neq 0, \\ O\left(k^{-1} \log (k)\right) & \text { otherwise. }\end{cases}
$$


Assumption (A.4) also yields $|H| \leq M \mathrm{Id}^{-q} \log (1 / x)$ on the open unit interval and thus the second term in (7) is $O\left(k^{q-1} \log (k)\right)$. It follows that

$$
T_{n}^{(1,1)}=\mu(W)+O\left(k^{q-1} \log (k)\right) .
$$

Besides, the well-known inequality $|\log (1+x)-x| \leq x^{2} / 2$, valid for all nonnegative $x$ together with (A.4) show that $\left|T_{n}^{(1,2)}\right|$ is bounded by

$$
\left|T_{n}^{(1,2)}\right| \leq \frac{M}{2 \log ^{2}(n / k)} \frac{1}{k-1} \sum_{i=1}^{k-1}(i / k)^{-q} \log ^{2}(k / i)=O\left(\frac{1}{\log ^{2}(n / k)}\right),
$$

since the above Riemann sum converges to a finite integral. Collecting (8) and (9) gives the result.

Second, we focus on the random term $T_{n}^{(2, \rho)}$.

Lemma 4 Let $\xi$ be standard Gaussian random variable. Under (A.1)-(A.4), the following expansion hold for all non-positive $\rho$ :

$$
T_{n}^{(2, \rho)} \stackrel{d}{=} \frac{\mu(W)}{E_{n-k+1, n}}\left\{1+\frac{\sigma(W)}{\mu(W)} k^{-1 / 2} \xi\left(1+o_{P}(1)\right)+O_{P}\left(\frac{1}{\log (n / k)}\right)+O_{P}\left(\|\varepsilon\|_{n, \infty}\right)\right\} .
$$

Proof : Note that $T_{n}^{(2, \rho)}$ can be written as the sum

$$
\frac{1}{k-1} \sum_{i=1}^{k-1} \varepsilon_{k-i, n} K_{\rho}\left(1+\frac{F_{i, k-1}}{E_{n-k+1, n}}\right)+\frac{1}{k-1} \sum_{i=1}^{k-1} J(i / k) K_{\rho}\left(1+\frac{F_{i, k-1}}{E_{n-k+1, n}}\right) .
$$

Since $0 \leq K_{\rho}(1+x) \leq x$ for all nonnegative $x$, the absolute value of the first term is bounded by

$$
\|\varepsilon\|_{n, \infty} \frac{1}{k-1} \sum_{i=1}^{k-1} K_{\rho}\left(1+\frac{F_{i, k-1}}{E_{n-k+1, n}}\right) \leq\|\varepsilon\|_{n, \infty} \frac{1}{k-1} \sum_{i=1}^{k-1} \frac{F_{i, k-1}}{E_{n-k+1, n}},
$$

which has the same distribution as

$$
\frac{\|\varepsilon\|_{n, \infty}}{E_{n-k+1, n}} \frac{1}{k-1} \sum_{i=1}^{k-1} F_{i}=\frac{1}{E_{n-k+1, n}} O_{P}\left(\|\varepsilon\|_{n, \infty}\right),
$$

from the law of large numbers. The second term of (10) can be expanded as

$$
\begin{array}{r}
\frac{1}{E_{n-k+1, n}} \frac{1}{k-1} \sum_{i=1}^{k-1} J(i / k) F_{i, k-1}+\frac{1}{k-1} \sum_{i=1}^{k-1} J(i / k)\left\{K_{\rho}\left(1+\frac{F_{i, k-1}}{E_{n-k+1, n}}\right)-\frac{F_{i, k-1}}{E_{n-k+1, n}}\right\} \\
=: \frac{T_{n}^{(2, \rho, 1)}}{E_{n-k+1, n}}+T_{n}^{(2, \rho, 2)} .
\end{array}
$$

Now, (A.3) and (A.4) imply that the L-statistics $T_{n}^{(2, \rho, 1)}$ satisfies the conditions of [21] and thus is asymptotically Gaussian. More precisely, we have

$$
T_{n}^{(2, \rho, 1)} \stackrel{d}{=} \mu(W)+\sigma(W) k^{-1 / 2} \xi\left(1+o_{P}(1)\right) .
$$

The upper bound on $T_{n}^{(2, \rho, 2)}$ is obtained by remarking that $\left|K_{\rho}(1+x)-x\right| \leq(1-\rho) x^{2} / 2$ for all nonnegative $x$. It follows that $T_{n}^{(2, \rho, 2)}$ is bounded above by

$$
\frac{1}{k-1} \sum_{i=1}^{k-1}|J(i / k)|\left|K_{\rho}\left(1+\frac{F_{i, k-1}}{E_{n-k+1, n}}\right)-\frac{F_{i, k-1}}{E_{n-k+1, n}}\right| \leq \frac{1-\rho}{2 E_{n-k+1, n}^{2}} \frac{1}{k-1} \sum_{i=1}^{k-1}|J(i / k)| F_{i, k-1}^{2} .
$$


Now, $E_{n-k+1, n}$ is equivalent to $\log (n / k)$ in probability and

$$
\frac{1}{k-1} \sum_{i=1}^{k-1}|J(i / k)| F_{i, k-1}^{2}=O_{P}(1)
$$

from the results of [21] on L-statistics. Thus

$$
T_{n}^{(2, \rho, 2)}=\frac{1}{E_{n-k+1, n}} O_{P}\left(\frac{1}{\log (n / k)}\right),
$$

and then collecting (11) and (12), the second term of (10) is

$$
\frac{1}{E_{n-k+1, n}}\left(\mu(W)+\sigma(W) k^{-1 / 2} \xi\left(1+o_{P}(1)\right)+O_{P}\left(\frac{1}{\log (n / k)}\right)\right),
$$

and the result follows.

We are now in position to prove Theorem 1 and Corollary 2 ,

Proof of Theorem 1. From Lemma 1, $k^{1 / 2}\left(\hat{\theta}_{n}(\alpha)-\theta\right)$ has the same distribution as

$$
\theta k^{1 / 2}\left(\frac{T_{n}^{(2,0)}}{T_{n}^{(1)}}-1\right)+k^{1 / 2} b\left(E_{n-k+1, n}\right) \frac{T_{n}^{(2, \rho)}}{T_{n}^{(1)}}\left(1+o_{P}(1)\right) .
$$

Now, $E_{n-k+1, n}$ is equivalent to $\log (n / k)$ in probability which is also equivalent to $\log (n)$, see Lemma 5.1 in [13]. Since $|b|$ is regularly varying (see [15]), $b\left(E_{n-k+1, n}\right.$ ) is equivalent to $b(\log (n))$ in probability. As a consequence, $k^{1 / 2}\left(\hat{\theta}_{n}(\alpha)-\theta\right)$ has the same distribution as

$$
\theta k^{1 / 2}\left(\frac{T_{n}^{(2,0)}}{T_{n}^{(1)}}-1\right)+k^{1 / 2} b(\log (n)) \frac{T_{n}^{(2, \rho)}}{T_{n}^{(1)}}\left(1+o_{P}(1)\right) .
$$

Let us consider the first term of this sum. Lemma 3, Lemma 4 and condition (4) entail that, for all non-positive $\rho$, the ratio $T_{n}^{(2, \rho)} / T_{n}^{(1)}$ has the same distribution as

$$
\frac{\log (n / k)}{E_{n-k+1, n}}\left\{1+\frac{\sigma(W)}{\mu(W)} k^{-1 / 2} \xi\left(1+o_{P}(1)\right)\right\} .
$$

Now, Lemma 1 in [16] asserts a central limit theorem for order statistics of an exponential sample, and thus

$$
\frac{\log (n / k)}{E_{n-k+1, n}} \stackrel{d}{=} 1+O_{P}\left(\frac{k^{-1 / 2}}{\log (n)}\right) .
$$

Consequently, the first term of (13) converges in distribution to $\mathcal{N}\left(0, \theta^{2} \sigma^{2}(W) / \mu^{2}(W)\right)$. We also have that the second term of (13) converges to $\lambda$ in probability and the result is proved.

Proof of Corollary 2, First remark that (6) can be rewritten as

$$
\hat{\theta}_{n}^{Z}=\sum_{i=1}^{k-1} \alpha_{i, n}^{Z}\left(\log \left(X_{n-i+1, n}\right)-\log \left(X_{n-k+1, n}\right)\right) / \sum_{i=1}^{k-1} \alpha_{i, n}^{Z}(\log \log (n / i)-\log \log (n / k)),
$$

where

$$
\begin{aligned}
\alpha_{i, n}^{Z} & =\log (n / k)\left(\log \log (n / i)-\zeta_{n}\right) \\
& =\log (n / k)\left(\log \left(1+\frac{\log (k / i)}{\log (n / k)}\right)-\tau_{n}\right) \\
& =\log (k / i)+O\left(\frac{\log ^{2}(k)}{\log (n)}\right)-\log (n / k) \tau_{n}, \\
& =\log (k / i)-1+O\left(\frac{\log ^{2}(k)}{\log (n)}\right)+O\left(\frac{\log (k)}{k}\right),
\end{aligned}
$$


uniformly on $i=1, \ldots, k$ with Lemma 2, Therefore, we have $\alpha_{i, n}^{Z}=W(i / k)+\varepsilon_{i, n}$ with $W(x)=-(\log (x)+1)$ and $\varepsilon_{i, n}=O\left(\log ^{2}(k) / \log (n)\right)+O(\log (k) / k)$, uniformly on $i=1, \ldots, k$. Then, it is easy to check that $W$ satisfies conditions (A.3) and (A.4) and that $\mu(W)=1$ and $\sigma^{2}(W)=2$.

\begin{tabular}{|c|c|c|c|c|}
\hline & $1-F(x)$ & $\theta$ & $b(x)$ & $\rho$ \\
\hline \hline Weibull \\
$\mathcal{W}(\alpha, \lambda)$ & $\exp \left(-(x / \lambda)^{\alpha}\right)$ & $1 / \alpha$ & 0 & $-\infty$ \\
\hline $\begin{array}{c}\text { Extended Weibull } \\
\mathcal{E} W(\tau, \beta, \gamma)\end{array}$ & $r(x) \exp \left(-\beta x^{\tau}\right)$ & $1 / \tau$ & $-\frac{\gamma}{\tau^{2}} \frac{\log x}{x}$ & -1 \\
\hline $\begin{array}{c}\text { Gaussian } \\
\mathcal{N}\left(\mu, \sigma^{2}\right)\end{array}$ & $\frac{1}{\left(2 \pi \sigma^{2}\right)^{1 / 2}} \int_{x}^{\infty} \exp \left(-\frac{(t-\mu)^{2}}{2 \sigma^{2}}\right) d t$ & $1 / 2$ & $\frac{1}{4} \frac{\log x}{x}$ & -1 \\
\hline $\begin{array}{c}\text { Gamma } \\
\Gamma(\beta, \alpha)\end{array}$ & $\frac{\beta^{\alpha}}{\Gamma(\alpha)} \int_{x}^{\infty} t^{\alpha-1} \exp (-\beta t) d t$ & 1 & $(1-\alpha) \frac{\log x}{x}$ & -1 \\
\hline $\begin{array}{c}\text { Benktander II } \\
\mathcal{B}(\alpha, \tau)\end{array}$ & $x^{\tau-1} \exp \left(-\frac{\alpha}{\tau} x^{\tau}\right)$ & $1 / \tau$ & $\frac{(1-\tau)}{\tau^{2}} \frac{\log x}{x}$ & -1 \\
\hline $\begin{array}{c}\text { Logistic } \\
\mathcal{L}\end{array}$ & $\frac{2}{1+\exp x}$ & 1 & $-\frac{\log 2}{x}$ & -1 \\
\hline $\begin{array}{c}\text { Extreme Value } \\
\mathcal{E} V D(\mu)\end{array}$ & $1-\exp (-\exp (\mu-x))$ & 1 & $-\frac{\mu}{x}$ & -1 \\
\hline
\end{tabular}

Table 1: Some Weibull tail-distributions 


\section{References}

[1] Arnold, B.C, Balakrishnan, N., Nagaraja H.N., (1992), A First course in order statistics, Wiley and sons.

[2] Bain, L., Engelhardt, M., (1991), Statistical analysis of reliability and life-testing models. Theory and methods. 2nd ed., Statistics: Textbooks and Monographs Series, 115, Marcel Dekker, Inc.

[3] Beirlant, J., Dierckx, G., Goegebeur, Y., Matthys, G., (1999), Tail index estimation and an exponential regression model, Extremes, 2, 177-200.

[4] Beirlant J., Broniatowski, M., Teugels, J.L., Vynckier, P. (1995), The mean residual life function at great age: Applications to tail estimation, Journal of Statistical Planning and Inference, 45, 21-48.

[5] Beirlant, J., Teugels, J.L., (1992), Modeling large claims in non-life insurance. Insurance: Math. Econom., 11, 17-29.

[6] Beirlant, J., Teugels, J., Vynckier, P., (1996), Practical analysis of extreme values, Leuven university press, Leuven.

[7] Berred, M., (1991), Record values and the estimation of the Weibull tail-coefficient. Comptes-Rendus de l'Académie des Sciences t. 312, Série I, 943-946.

[8] Bingham, N.H., Goldie, C.M., Teugels, J.L., (1987), Regular variation, Encyclopedia of Mathematics and its Applications, 27, Cambridge University Press.

[9] Broniatowski, M., (1993), On the estimation of the Weibull tail coefficient, Journal of Statistical Planning and Inference, 35, 349-366.

[10] Csörgö, S., Deheuvels, P., Mason, D., (1985), Kernel estimates of the tail index of a distribution, The Annals of Statistics, 13, 1050-1077.

[11] Embrechts, P., Klüppelberg, C., Mikosch, T., (1997), Modelling extremal events, Springer.

[12] Galambos, J., (1987), The Asymptotic theory of extreme order statistics, R.E. Krieger publishing company.

[13] Gardes, L., Girard, S., (2006), Comparison of Weibull tail-coefficient estimators, REVSTAT - Statistical Journal, 4, 373-188.

[14] Gardes, L., Girard, S., (2008), Estimation of the Weibull tail-coefficient with linear combination of upper order statistics, Journal of Statistical Planning and Inference, 138, $1416-1427$.

[15] Geluk, J.L., de Haan, L., (1987), Regular Variation, Extensions and Tauberian Theorems. Math Centre Tracts, 40, Centre for Mathematics and Computer Science, Amsterdam.

[16] Girard, S. (2004), A Hill type estimate of the Weibull tail-coefficient, Communication in Statistics - Theory and Methods, 33, 205-234.

[17] Häusler, E., Teugels, J.L. (1985), On asymptotic normality of Hill's estimator for the exponent of regular variation, The Annals of Statistics, 13, 743-756.

[18] Hill, B.M., (1975), A simple general approach to inference about the tail of a distribution, The Annals of Statistics, 3, 1163-1174.

[19] Klüppelberg, C., Villaseñor, J.A., (1993), Estimation of distribution tails - a semiparametric approach, Deutschen Gesellschaft für Versicherungsmathematik, XXI(2), 213235. 
[20] Kratz, M., Resnick, S., (1996), The QQ-estimator and heavy tails, Stochastic Models, 12, 699-724.

[21] Mason, D.M., (1981), Asymptotic normality of linear combinations of order statistics with a smooth score function, Annals of Statistics, 9(4), 899-908.

[22] Schultze, J., Steinebach, J., (1996), On least squares estimates of an exponential tail coefficient, Statistics and Decisions, 14, 353-372.

[23] Viharos, L. (1999), Weighted least-squares estimators of tail indices, Probability and Mathematical Statistics, 19, 249-265. 